stimulates growth and actually boosts overall wealth. At least, that's the conclusion of two of the models - one developed at the University of Cambridge, UK, and the other at the Fondazione Eni Enrico Mattei, a centre for sustainable-development research in Italy. These models suggest that stabilization policies would give an added boost to global GDP of up to $1.7 \%$ over 100 years. They assume such climate policies will bring about side benefits, such as increased investment in new technologies.

Ottmar Edenhofer, an economist at the Potsdam Institute for Climate Impact Research in Germany who edited the issue along with Grubb and others, says the new estimates of lost global GDP are significantly lower than previous ones, which put the range at $3-15 \%$. They suggest the price will be a lot lower, agrees Terry Barker, an economist who helped developed the Cambridge model, especially as costs will be spread over 100 years.

The models are likely to influence the next report from the Intergovernmental Panel on Climate Change, due for publication next year. The authors hope the results will then filter through to governments. They say the cheapest stabilization route can only be achieved if industries are given a strong signal that carbon emissions will continue to be restricted - and that means the United States must join a future version of the Kyoto Protocol. Europe also needs to do more, say the authors, particularly in terms of investment in energy technologies, where it lags behind the United States.

But some economists are wary of the results. Jae Edmonds of the Pacific Northwest National Laboratory in Richland, Washington, describes the models as a valuable "intellectual experiment". But he questions the fact that most of the models emphasize learning-by-doing - a process by which technologies become cheaper as industry learns how to produce them more efficiently.

Edmonds points out, for example, that many advances in hybrid vehicles have been made possible by developments in areas outside the automotive industry. It is hard to distinguish these advances from those due to learning-by-doing or research by automotive firms. If advances are attributed exclusively to one mechanism, Edmonds warns that the benefits of the process could be exaggerated and costs underestimated: "We don't necessarily have a good handle on how important different factors are and how they interact." Jim Giles

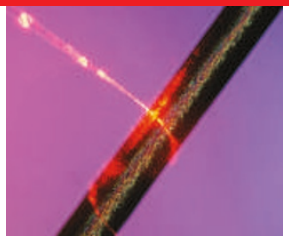

PHYSICS NEWS

Get breaking news on the physical sciences from our website. www.nature.com/news/ channels/physicalsciences.htm

\title{
TOP FIVE IN PHYSICS
}

Are you working on the hottest topic in your field? Many scientists may think so, but it has been a tough assertion to prove - until now, that is. A German physicist has devised a way of answering the 'Hot or not?' question for his discipline. If it stands up to scrutiny, it could be used to rate topics across the sciences. In physics, the results show that hotness - measured by a parameter known as $m$ - correlates well with the promise of future wealth... and that promise is greatest in nanotechnology.

\subsection{Carbon nanotubes}

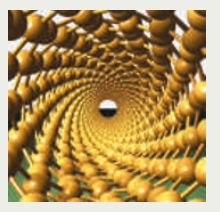

Super-strong materials and blisteringly fast electronic circuits: the potential applications of these tiny carbon tubes, discovered in 1991, are so enticing that everyone is pouring money into the field.

\subsection{Nanowires}

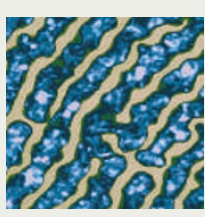

Less well studied than nanotubes, but the possible uses are similar. Nanowires could eventually prove more useful than nanotubes, because their chemistry is easier to tailor and they can be used to create nano-sized lasers.

\subsection{Quantum dots}

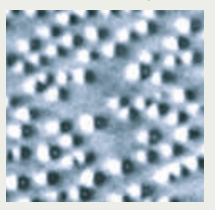

Another nanotechnology with a huge range of potential applications. These tiny specks of semiconductor material, measuring as little as a few nanometres across, have already been used to create dyes for cell biologists and new kinds of laser. Physicists hope they might one day form the basis of a quantum computer.

\subsection{Fullerenes}

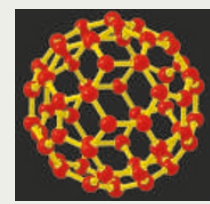

These spheres of carbon atoms are attracting significant research interest. But the latest ranking rewards newness, so the topic may have slipped down the list because it predates nanotubes by around six years. The discovery of fullerenes earned a Nobel prize and spawned studies of numerous potential uses, such as drug delivery agents.

\subsection{Giant magnetoresistance}

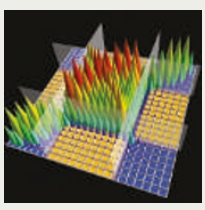

Not a new topic, but still hot because of its economic importance. Modern hard disk drives were made possible by the discovery of giant magnetoresistant materials, which show marked falls in electrical resistance - more than around $5 \%$ - when a magnetic field is applied. Researchers are now aiming to make hard disks even more powerful.

\section{How the topics were ranked}

The ranking is an extension of a recently proposed system for rating the research output of individual scientists. The $h$ index uses the highest number of papers a scientist has published that have each received at least that number of citations: for example, a researcher with an $h$ of 50 has written 50 papers that have each had at least 50 citations (see Nature 436, 900; 2005). The index has attracted interest from bibliometricians, but was first described only last year and has yet to be studied in depth. Michael Banks, a PhD student at the Max
Planck Institute for Solid State Research in Stuttgart, Germany, totted up the $h$ index for physics topics, rather than people, and then calculated the parameter $m$ by dividing by the number of years over which the papers involved had been published (www.arxiv.org/abs/ physics/0604216).

Jim Giles

Do you agree with the winners? Tell us what you think on our news blog

|blogs.nature.com/news/blog/2006/05/

top_five_in_physics.html 